\title{
Erratum to: HER2 Ile655Val polymorphism contributes to breast cancer risk: evidence from 27 case-control studies
}

Su Lu $\cdot$ Zhanwei Wang · Hong Liu •

Xishan Hao

Published online: 7 October 2010

(C) Springer Science+Business Media, LLC. 2010

Erratum to: Breast Cancer Res Treat

DOI 10.1007/s10549-010-0886-z

Authors would like to add the following Acknowledgements:
Acknowledgments This work is supported by the Innovation Funding for graduates of Tianjin Medical University, third phase of the 211 Project for Higher Education (No. 2009GSI09).

The online version of the original article can be found under doi:10.1007/s10549-010-0886-z.

S. Lu $\cdot$ H. Liu $\cdot$ X. Hao $(\square)$

Key Laboratory of Breast Cancer Prevention and Therapy,

Tianjin Medical University, Ministry of Education,

30006 Tianjin, China

e-mail: xishan_hao@yahoo.com

S. Lu $\cdot$ H. Liu $\cdot$ X. Hao

Key Laboratory of Cancer prevention and Therapy,

30006 Tianjin, China

S. Lu $\cdot$ H. Liu $(\bowtie) \cdot X$. Hao

Second Department of Breast Tumor, Tianjin Medical

University Cancer Institute and Hospital, 300060 Tianjin, China

e-mail: liuh@medmail.com.cn

\section{Z. Wang}

Department of Epidemiology and Biostatistics, Nanjing Medical

University, 210029 Nanjing, China 